\title{
Effect of accelerated storage on microencapsulated kenaf seed oil.
}

\begin{abstract}
In order to improve the quality and protect against degradation, kenaf (Hibiscus cannabinus L.) seed oil was microencapsulated by using spray drying. The microencapsulated kenaf seed oil (MKSO) was then stored at $65{ }^{\circ} \mathrm{C}$ for 24 days, the changes of fatty acids and bioactive compounds were examined every six days. Bulk (unencapsulated) kenaf seed oil was used as a control and was compared to the MKSO. The fatty acids and phytosterols compositions were determined by using gas chromatography, while tocopherols and phenolic acids of microencapsulated kenaf seed oil were determined by using high performance liquid chromatography. The results showed that there was a significant decrease $(p<0.05)$ in bioactive compounds in kenaf seed oil while the bioactive compounds in MKSO were maintained in a stable condition upon accelerated storage. Microencapsulation was shown to protect kenaf seed oil against oxidation, as well as preventing the degradation and/or loss of bioactive compounds in kenaf seed oil.
\end{abstract}

Keyword: Hibiscus cannabinus seed oil; Spray drying; Fatty acids; Phytosterols; Tocopherols; Phenolic acids; Gas chromatography (GC); High performance liquid chromatography (HPLC) 